\title{
Hot Topic
}

\section{Severe Type of COVID-19: Pathogenesis, Warning Indicators and Treatment}

\author{
SHI Ke ${ }^{1,2}$, LIU Yao ${ }^{1}$, ZHANG Qun ${ }^{1}$, RAN Chong-ping ${ }^{1}$, HOU Jie ${ }^{1,2}$, ZHANG Yi $^{1}$, and WANG Xian-bo ${ }^{1}$
}

\begin{abstract}
Coronavirus disease 2019 (COVID-19), caused by severe acute respiratory syndrome coronavirus 2 , is a major public health issue. The epidemic is unlikely to be contained until the global launch of safe and effective vaccines that could prevent serious illnesses and provide herd immunity. Although most patients have mild flu-like symptoms, some develop severe illnesses accompanied by multiple organ dysfunction. The identification of pathophysiology and early warning biomarkers of a severe type of COVID-19 contribute to the treatment and prevention of serious complications. Here, we review the pathophysiology, early warning indicators, and effective treatment of Chinese and Western Medicine for patients with a severe type of COVID-19.
\end{abstract}

KEYWORDS coronavirus disease 2019, pathogenesis, indicators, treatment, Chinese medicine

The coronavirus disease 2019 (COVID-19) pandemic is a major public health event in the world, which poses a great threat to people health and the economy. Clinical manifestations of patients with COVID-19 include fever, cough, fatigue, acute respiratory distress syndrome (ARDS), sepsis, and even death. ${ }^{(1,2)}$ China has contained spread of the epidemic attributing to the intervention strategies of early detection, isolation, and treatment. The vaccine is a key weapon against COVID-19 because it can prevent hospitalization and severe diseases, and may provide herd immunity. ${ }^{(3,4)}$ With the understanding of the protein constituents and genomic sequence of severe acute respiratory syndrome coronavirus 2 (SARS-CoV-2), several vaccines have been used for humans. ${ }^{(4,5)}$ More than 60 vaccines are in the stage of clinical development. ${ }^{(4)}$

Although most patients with COVID-19 present with mild and moderate syndrome, severe infection can cause rapid progression, ARDS, coagulation dysfunction, and multiple organ failure. ${ }^{(6,7)}$ Severe type of COVID-19 often occurs in elderly patients with comorbidities, ${ }^{(8)}$ which bring burden to medical resources and medical staff. Early identification and effective management strategy are of great significance to reasonable resource allocation and reduction of mortality. ${ }^{(9,10)}$ Thus, how to prevent occurrence of severe type of COVID-19 is a noteworthy problem. This paper summarizes the potential pathogenesis and early warning indicators of severe type of COVID-19. Besides, we highlight efficient therapeutic approaches of Western medicine and Chinese medicine (CM).

\section{Pathogenesis}

\section{Pathophysiology}

Severe type of COVID-19 might manifest as substantial damage to multiple organs (Figure 1). The pathophysiology of severe SARS-CoV-2 infection includes virus damage, endotheliopathy, and thrombosis, immune-inflammatory response, and renin-angiotensinaldosterone (RAAS) system disorder (Figure 2).

\section{Direct Viral Damage}

Through the S protein, SARS-CoV-2 binds to the angiotensin-converting enzyme 2 (ACE2) receptor. ${ }^{(11,12)}$ The cleavage of $S$ protein by transmembrane protease serine 2 (TMPRSS2) makes coronavirus gain access to host cells. ${ }^{(11,12)}$ ACE2 and TMPRSS2 are abundantly expressed in pneumocytes, cardiac myocytes, small intestineepithelial cells, and renal proximal tubules, and direct viral injury is a possible mechanism in severe COVID-19..$^{(12-15)}$

Viral replication occurs chiefly in type II pneumocytes, leading to continued destruction of the alveolar wall. ${ }^{(16)}$ In

CThe Chinese Journal of Integrated Traditional and Western Medicine Press and Springer-Verlag GmbH Germany, part of Springer Nature 2021

1. Center of Integrative Medicine, Beijing Ditan Hospital, Capital Medical University, Beijing (100015), China; 2. Department of Gastroenterology, Dongzhimen Hospital, Beijing University of Chinese Medicine, Beiijing (100007), China

Correspondence to: Prof. WANG Xian-bo, E-mail: wangxb@ ccmu.edu.cn

DOI: https://doi.org/10.1007/s11655-021-3313-x 


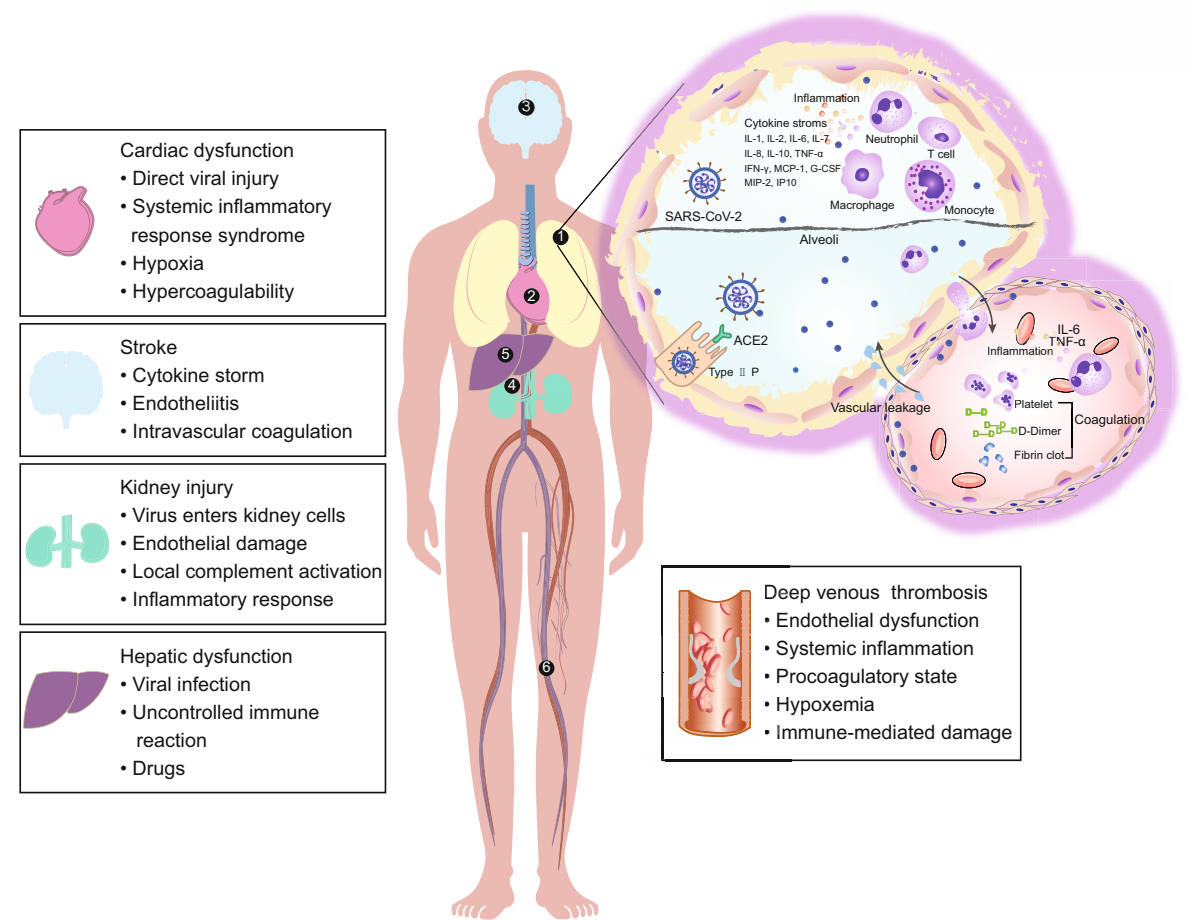

Figure 1. Potential Mechanisms of Multiple Organ Dysfunction Induced by Severe Type of COVID-19

Notes: Pathophysiological characteristics of lung: endothelial injury, inflammation, and thrombosis. Other organ dysfunction, including heart, brain, kidney, liver, and deep vein, are also summarized. IL: interleukin; TNF- $\alpha$ : tumor necrosis factor-alpha; IFN $\gamma$ : interferon gamma; MCP: monocyte chemoattractant protein; G-CSF: granulocyte-colony stimulating factor; MIP: macrophage inflammatory protein; IP10: IFN $\gamma$-inducible protein 10; SARS-CoV-2: severe acute respiratory syndrome coronavirus-2; Type II P: type II pneumocyte; ACE2: angiotensin-converting enzyme 2

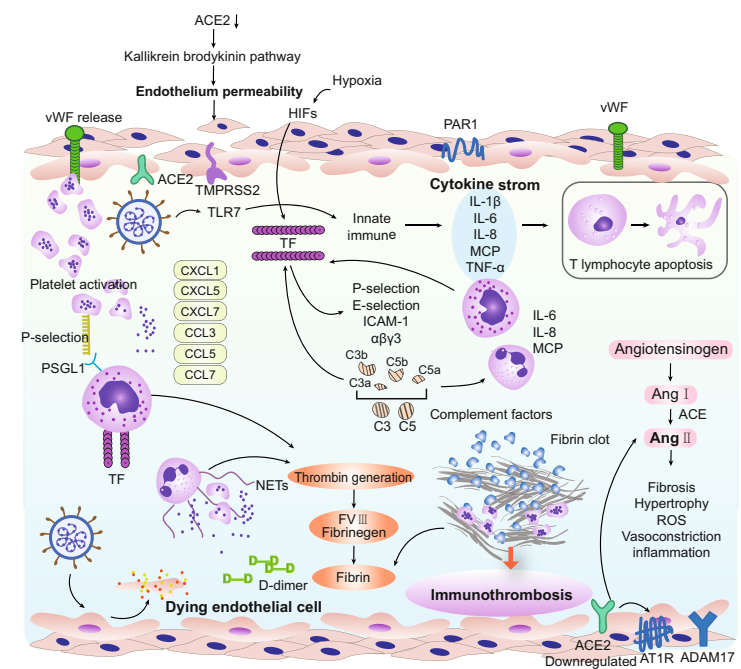

Figure 2. Pathophysiology for Patients with Severe Type of COVID-19

Notes: ACE2: angiotensin-converting enzyme 2; Ang: angiotensin; ADAM-17: a disintegrin and metalloproteinase 17; AT1R: angiotensin 1 receptors; COVID-19: coronavirus disease 2019; CXCL: CXC-chemokine ligand; CCL: chemokine ligand; IL: interleukin; ICAM: intercellular adhesion molecule; HIF: hypoxia-inducible factor; MCP: monocyte chemoattractant protein; NETs: neutrophil extracellular traps; PSGL1: P-selectin glycoprotein ligand 1; PAR1: proteinase activated receptor 1; RAAS: renin-angiotensin-aldosterone system; ROS: reactive oxygen species; SARS-CoV-2: severe acute respiratory syndrome coronavirus-2; TMPRSS2: transmembrane protease serine 2; TLR: toll-like receptor; TNF- $\alpha$ : tumor necrosis factoralpha; TF: tissue factor; vWF: von Willebrand factor multimers the late stage of disease, SARS-CoV-2 is mainly replicated in the lower respiratory tract, accompanied by ARDS in severe cases. ${ }^{(17,18)}$ Numerous studies have indicated that SARS-CoV-2 is located in endothelial cells (ECs) in sections of the lungs, kidneys, and intestine. ${ }^{(12,19)}$

\section{Endotheliopathy and Thrombosis}

The key points of thrombosis are endothelial dysfunction, complement activation, immune response initiation, cytokine infiltration, platelet-leukocyte aggregation, hypoxia, and thrombin generation. ${ }^{(20,21)}$

Indeed, complement is an important component of innate immunity to SARS-CoV-2. ${ }^{(20)}$ The recognition of the virus by toll-like receptor 7 (TLR7) may lead to innate immune hyperactivation. ${ }^{(22)}$ Unrestrained activation of the complement increases endothelial permeability, facilitates the recruitment of monocytes and neutrophils, and mediates generalized microvascular injury, which culminates in immunothrombosis in severe COVID-19 cases. ${ }^{(23,24)}$

The activation of platelets releases chemokines, including CXC-chemokine ligand 1 (CXCL1), CXCL5, chemokine ligand 3 (CCL3), and CCL5, ${ }^{(25)}$ which facilitate 
leukocyte recruitment to sites of vascular injury. ${ }^{(26)}$ Activated platelets promote neutrophils to discharge eneutrophil extracellular traps (NETs). ${ }^{(27,28)}$ Although NETs are beneficial inensnaring pathogens, excessive NETs correlate with proinflammatory cytokine production, activation of the coagulation pathway, and multiple organ damage and death. ${ }^{(29)}$ Platelet degranulation, leukocyteplatelet interactions, and platelet C3 release in turn stimulate NETs. ${ }^{(30,31)}$

Patients with severe type of COVID-19 have been demonstrated to have increased interleukin (IL)-1 $\beta$, IL-6, monocyte chemoattractant protein-1 (MCP-1), interferon gamma (IFN $\gamma$ ), IFN $\gamma$-inducible protein 10 (IP10), macrophage inflammatory protein $1 \alpha$ (MIP-1 $\alpha$ ), and tumor necrosis factor (TNF)- $\alpha .^{\left({ }^{(32-34)}\right.}$ These inflammatory cytokines facilitate vascular endotheliopathy, leading to the release of von Willebrand factor and overexpression of tissue factor (TF) and fibrinogen. ${ }^{(35-37)}$ These factors contribute substantially to increased mortality due to COVID-19 and affect other organs, leading to ischemic stroke, pulmonary embolisms, cardiac injury, acute kidney injury, and deep vein thrombosis (DVT). ${ }^{(18,20,37-40)}$

Inflammation-induced endothelial injury can lead to abundant plasminogen activator infiltration. ${ }^{(23,41)}$ Thrombin, via proteinase activated receptor 1 , promotes platelet activation, enhances the release of inflammatory factors, and activates the coagulation pathway. ${ }^{(42)}$ In addition, hypoxia induces upregulation of hypoxia-inducible factor 1 signaling pathway, contributing to TF expression, vasoconstriction, inflammation, and thrombosis. ${ }^{(43,44)}$

\section{Immune Inflammatory Response}

Overactivation of innate immunity-induced cytokine storm is a feature of severe COVID-19. ${ }^{(45)}$ Activated macrophages and neutrophils play a prominent role in the hyperinflammatory response and release cytokines and chemokines, including IL-1 $\beta$, IL-6, IL-7, granulocyte-colony stimulating factor (G-CSF), IFN- $\gamma, \mathrm{MCP}-1, \mathrm{MIP}-1 \alpha, \mathrm{IP} 10$, TNF- $\alpha$, and C-reactive protein (CRP)..$^{(2,45-48)}$ Furthermore, a prominent feature of severe SARS-CoV-2 infection is lymphocytopenia, which is associated with adverse outcomes. ${ }^{(49,50)}$ Because of massive T-cell lymphodepletion and dysregulation of the immune response, a number of cytokines are released. Excessive IL-6 can induce TF expression on mononuclear cells, upregulation of adhesion molecules including $\mathrm{P}$ - and E-selectin and integrin $\alpha$ and $\beta 3$, and subsequently act to enhance coagulation and thrombin generation. ${ }^{(51,52)}$

\section{Disorders of RAAS}

Dysregulation of the RAAS is involved in the pathophysiological mechanism of severe COVID-19associated tissue damage. ${ }^{(53)}$ ACE2 serves a counterbalancing role in the RAAS, including bloodpressure regulation, fibrosis, and inflammation. ${ }^{(54,55)} \mathrm{ACE}$ promotes the conversion of angiotensin I (Ang I ) to Ang II. ${ }^{(56)}$ SARS-CoV-2 impairs the activity of ACE2, resulting in an increase in Ang II with increased angiotensin 1 receptor (AT1R) activation. ${ }^{(55,57-59)}$ Subsequently, the activation of a disintegrin and metalloproteinase 17 resulted in ACE2 membrane shedding, RAAS overactivation, and a proinflammatory cascade. ${ }^{(60)}$ Additionally, downregulation of ACE2 expression by SARS-CoV-2 infection reduced ACE2 and activated kallikrein-bradykinin pathway, increasing risk of vascular leakage, leading to further pulmonary edema. ${ }^{(41)}$ Abnormal activation of ACE/Ang II axis leads to pulmonary vasoconstriction, inflammation, fibrosis, hypertrophy, and accumulation of reactive oxygen species. ${ }^{(12,58)}$

\section{Pathogenesis of CM}

COVID-19 belongs to the category of "plague" in CM theory, which is caused by the cold-dampness epidemic virus. ${ }^{(61,62)}$ This disease is located in Fei (Lung) and can affect Xin (Heart), Pi (Spleen), Gan (Liver), Shen (Kidney), and other organs. ${ }^{(62,63)}$ The basic pathogenesis is characterized by dampness, toxin, blood stasis, and closure. ${ }^{(64)}$

\section{Early Warning Indicators}

A pattern of general features, comorbidities, hematologic, biochemical, coagulation, immune, and inflammatory biomarkers have been identified in patients with severe type of COVID-19 that included factors predicting disease progression (Figure 3).

\section{General Features and Comorbidities}

Compared to patients with non-severe COVID-19, those with severe COVID-19 were older, male, and had more comorbidities. ${ }^{(65-68)}$ Regarding the underlying diseases associated with severe disease, the most recorded one was hypertension, followed by diabetes mellitus, cardiac diseases, kidney disease, chronic obstructive pulmonary disease (COPD), and malignant tumors ${ }^{(69-72)}$ Downregulated $\mathrm{PaO}_{2}: \mathrm{FiO}_{2}$ and $\mathrm{SpO}_{2} / \mathrm{FiO}_{2}$, and upregulated sepsis-related organ failure assessment are risk factors for death. ${ }^{(32,33,70)}$

\section{Hematologic Biomarkers}

The higher the white blood cell (WBC), neutrophil 


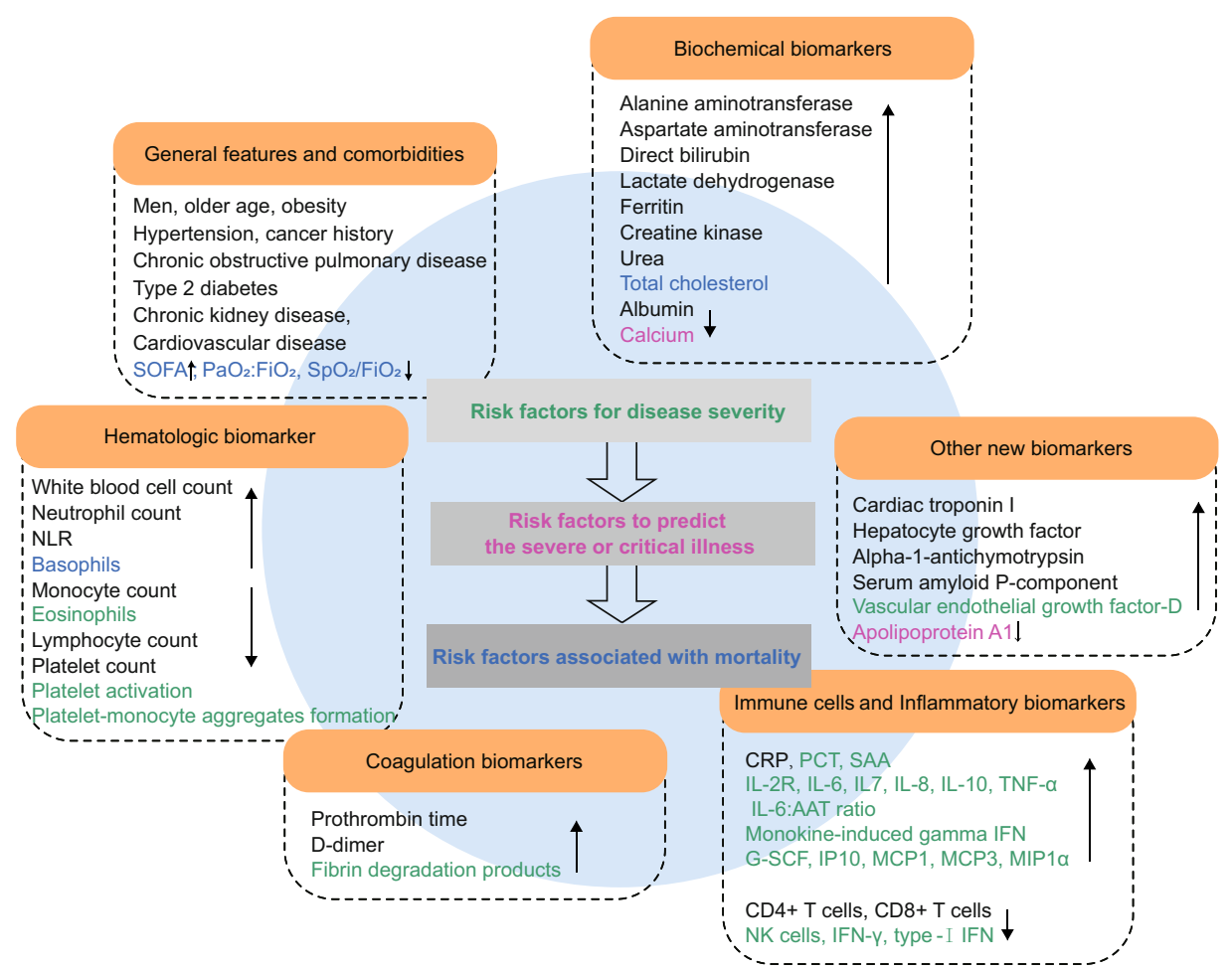

Figure 3. Early Warning Indicators in Severe Type of COVID-19 Progression

Notes: The text colors correspond to warning indicators for disease severity. Green text: severe illness; purple text: severe or critical illness; blue text: mortality; black text: possible predictive factors for severe, critical illness, and mortality. SOFA: sepsis-related organ failure assessment; $\mathrm{PaO}_{2}$ : partial pressure of oxygen; $\mathrm{FiO}_{2}$ : fraction of inspired $\mathrm{O}_{2} ; \mathrm{SpO}_{2}$ : pulse oxygen saturation; NLR: netrophillymphocty ratio; CRP: C-reactive protein; PCT: procalcitonin; SAA: serum amyloid protein A; IL: interleukin; TNF- $\alpha$ : tumor necrosis factor- $\alpha$; AAT: alpha-1 antitrypsin; IFN: interferon; G-SCF: granulocyte colony-stimulating factor; IP10: IFN- $\gamma$-induced protein 10; MCP: monocyte chemotactic protein; MIP1 $\alpha$ : macrophage inflammatory protein $1 \alpha$; NK cells: natural killer cells

count, neutrophil-lymphocyte ratio (NLR), and basophils, and the lower the platelet, lymphocyte (especially $\mathrm{CD}^{+}$ and $C D 8^{+} T$ cells), and monocyte count, the higher the mortality. ${ }^{(73)}$

\section{Biochemical Biomarkers}

Patients with severe illness more frequently showed increased alanine aminotransferase, aspartate aminotransferase, ferritin, and lactate dehydrogenase levels and lower levels of albumin. ${ }^{(74)}$ Lactate dehydrogenase (LDH), direct bilirubin, creatine kinase, urea, and calcium may serve as clinical predictors of severe or critical illness. ${ }^{(66,75,76)}$ Independent risk factors associated with mortality included hypercholesterolemia, liver dysfunction, kidney dysfunction, increased lactate dehydrogenase and ferritin, and low albumin levels. ${ }^{(77)}$

\section{Coagulation Biomarkers}

Patients with severe type of COVID-19 had higher D-dimer, fibrin degradation products, and prothrombin time than those with non-severe disease. Elevated D-dimer at initial presentation was predictive of coagulation-associated complications during hospitalization, critical illness, and death. ${ }^{(78)}$

\section{Immune and Inflammatory Biomarkers}

The inflammatory factors, mainly IL-6, increase substantially, which results indisease progression approximately $7-14$ days after onset. ${ }^{(79)} \mathrm{CRP}$ is related to disease progression and is a clinical predictor of severity and mortality. ${ }^{\left({ }^{80}\right)}$ Other relevated inflammatory biomarkers include procalcitonin, serum amyloid protein A, TNF- $\alpha$, IL-7, IL-9, IL-10, IL-6: alpha-1 antitrypsin ratio, monokineinduced IFN- $\gamma$, granulocyte-colony stimulating factor, IP10, MCP-1, MCP-3, and MIP-1 $\alpha .^{(66,81-85)}$ Patients with severe disease had a lower natural killer cell count, IFN- $\gamma$, and type- I IFNs than those with non-severe COVID-19. ${ }^{(86,87)}$

\section{Other New Biomarkers}

Markers of cardiac and muscle injury were elevated in patients with both severe and fatal COVID-19. Initial cardiac troponin I, hepatocyte growth factor, alpha-1antichymotrypsin, vascular endothelial growth factor-D, and serum amyloid P-component were substantially higher in patients with critical illness. ${ }^{(84,88)}$ 
Treatment

\section{Remdesivir}

Remdesivir, a nucleoside analog prodrug that impedes viral RNA synthesis, has been routinely used in the treatment of viral infections. ${ }^{(89,90)}$ In a randomized study conducted by Wang, et al, ${ }^{(91)}$ researchers found no statistically significant benefit of remdesivir when comparing placebo group with remdesivir group. Although not statistical significance was found, patients receiving remdesivir showed reduced duration of clinical symptoms compared to the control group within 10 days of symptom duration. Of note, a study by Siemieniuk, et al ${ }^{(92)}$ verified the effectiveness of remdesivir in shortening both the time to recovery and the duration of mechanical ventilation; however, the mortality benefit is uncertain. In view of the clinical effectiveness of remdesivir, the United States Food and Drug Administration (FDA) has authorized remdesivir for emergency use in the treatment of patients with severe disease.$^{(93)}$ Moreover, the drug has been recommended by the European Union. ${ }^{(94)}$

\section{Corticosteroids}

The National Institutes of Health COVID-19 Treatment Panel Guidelines recommended dexamethasone at a dose of $6 \mathrm{mg}$ once daily for up to 10 days for patients on mechanical ventilation and oxygen. ${ }^{(95)}$ Available evidence suggests that corticosteroids have been identified as the only drugs that reduce mortality and mechanical ventilation compared to standard treatment. ${ }^{(96)}$

A trial from the United Kingdom found that lowdose dexamethasone was associated with a $33 \%$ lower mortality rate in patients receiving invasive ventilation and $20 \%$ in those needing oxygen compared to the usual care group. ${ }^{(97)}$ In a multicenter study in hospitalized patients with ARDS patients administered dexamethasone had a 60-day mortality rate that was $15 \%$ lower than that of the control group. ${ }^{(98)} \mathrm{A}$ cohort study from China showed that methylprednisolone may reduce the mortality rate and has a significant clinical benefit in patients with COVID-19 with ARDS. ${ }^{(33)}$ Thus, the appropriate dosage of corticosteroids may maximize benefits and minimizing adverse effects.

\section{Anticoagulants}

Given that thrombotic complications confer a high death rate in patients with COVID-19, prophylactic or therapeutic anticoagulation (AC) is of critical importance and established in advance. ${ }^{(99)}$ Unfractionated heparin (UFH) or lowmolecular-weight heparin (LMWH) in hospitalized patients should be administered unless they have contraindications (active bleeding or severe thrombocytopenia). ${ }^{(10-102)}$

A retrospective analysis by Tang, et al ${ }^{(103)}$ found that patients administered heparin had a reduction in 28-day mortality. The use of AC mainly with LMWH markedly decreased the 28-day mortality in patients with severe COVID-19 with a sepsis-induced coagulopathy score of $\geqslant 4$ points or substantially increased D-dimer. Nevertheless, even with the use of LMWH or UFH, thrombotic events still occur. ${ }^{(7)}$ In a multicenter study, many patients with ARDS still experienced thrombotic events despite AC treatment, in which D-dimer and fibrinogen levels increased markedly. ${ }^{(104)}$ Thus, AC treatment needs to take into account the dose and coagulation characteristics of patients.

\section{Convalescent Plasma}

Convalescent plasma (CP) therapy has been applied in the treatment of SARS, Middle East Respiratory Syndrome, and H1N1 influenza, and has demonstrated efficacy. ${ }^{(105-107)}$ Shen, et al ${ }^{(108)}$ reported that 5 patients with COVID-19 with ARDS were successfully treated with $\mathrm{CP}$, as evidenced by improved clinical symptoms, and treatment contributed to the clearance of the virus. Another study reported that in 10 patients with severe illness, $200 \mathrm{~mL}$ CP could increase or maintain a high level of neutralizing antibodies without severe adverse effects. ${ }^{(109)}$ Moreover, clinical conditions rapidly improved within 3 days with increases in oxyhemoglobin saturation and lymphocyte counts. CP therapy offers a promising treatment option, and the United States FDA has authorized the use of plasma from convalescent patients to treat patients with severe COVID-19. ${ }^{(110)}$

\section{$\mathrm{CM}$}

In the severe type of COVID-19 patients, the common syndrome types include epidemic virus blocking the Lung, blazing of both qi and nutrient, and internal block and external collapse syndrome. ${ }^{(111)}$ The National Health Commission (NHC) of China has declared a combination of $\mathrm{CM}$ and Western medicine as a therapeutic regimen for COVID-19 and has published 8 versions of Diagnosis and Treatment Protocol. Several clinical studies showed that the combination of $\mathrm{CM}$ and Western medicine can improve the curative rate, shorten the average hospital stay, and reduce the number of cases from mild to severe. ${ }^{(112-114)}$ A propensity score-based analysis suggested that $\mathrm{CM}$ is effective in reducing the mortality rate in severe and critical illnesses. ${ }^{(115)} \mathrm{CM}$ combined with conventional treatment has a protective effect on patients with severe COVID-19, and can reduce fever time and mortality. ${ }^{(116)}$ A meta-analysis demonstrated that $\mathrm{CM}$ can 
effectively reduce severe cases, improve lung imaging manifestations, and shorten the length of hospital stay. ${ }^{(1)}$

Qingfei Paidu Decoction (清肺排毒汤, QFPD), a prescription optimized from Maxing Shigan Decoction (麻 杏石甘汤, MXSG), Shegan Mahuang Decoction (射千麻黄 汤), Xiaochaihu Decoction (小柴胡汤), and Wuling Powder (五苓散), has been listed in the 6th edition of the protocol as a recommended prescription. QFPD is suitable for patients of COVID-19 at all stages, with the total effective rate $92.09 \% .{ }^{(117,118)} \mathrm{A}$ multicenter cohort study manifested that early application of QFPD can improve nucleic acid negative conversion rate, reduce hospitalization time and mortality rate of patients. ${ }^{(119)}$

Huashi Baidu Decoction (化湿败毒方, HGBD) is applied to treat the epidemic virus blocking Lung syndrome. A result reported by Wuhan Jinyintan Hospital showed that compared with the Western medicine group, there was significantly better effect in pulmonary inflammation absorption, the mean duration of viral clearance, CRP level and serum iron in the severe patients treated with HGBD, Xuebijing Injection (血必净注 射液, XBJ), Xiyanping Injection (喜炎平注射液, XYP), and Shengmai Injection (参麦注射液, SM). ${ }^{(120)}$

Yindan Jiedu Granules (银丹解毒颗粒, YDJDG), an in-hospital prescription from Beijing Ditan Hospital, has showed good clinical effects in the treatment of mild, moderate, severe and critical patients with COVID-19. YDJDG is a combination and innovation based on MXSG, Qingwen Baidu Decoction (清瘟败毒方), and Tingli Dazao Xiefei Decoction (葶荕大來泻肺汤). In the YDJDG group, the proportion of recovered patients was remarkably higher than the lopinavir-ritonavir group. Compared with the control group, the average course of fever and pulmonary exudative lesions in the YDJDG group was remarkably shorter. ${ }^{(121)}$

Among the "Three CM and three formulas" recommended by the $\mathrm{NHC}, \mathrm{XBJ}$ is the only injection form. XBJ, XYP, Xingnaojing Injection (醒脑静注射液, XNJ), Reduning Injection (热毒宁注射液, RDN), Tanreqing Injection (痰热清注射液, TRQ), Shenfu Injection (参附注 射液, SF), and SM have been recommended for treating severe type of COVID-19 in the 6th and 7th edition of protocol. XBJ, XNJ, XYP, RDN, and TRQ indications are pattern of dual blaze of qi and nutrient. The indications of SF and SM are patients with pattern of internal block and external collapse. A case-control study showed that the body temperature and IL- 6 level of the patients treated with XBJ combined with Western medicine were significantly lower than the control group $(P<0.05) .{ }^{(122)}$ The details of CMs and injections for severe type of COVID-19 including compositions, function, indication, and on-going clinical researches are summarized in Appendix 1.

CM, with multi-targets and multi-pathways, has potential advantages in human body regulation. We analyzed the mechanism of $\mathrm{CM}$ in the treatment of severe type of COVID-19: (1) Direct inhibition of viruses. Emerging evidence suggested that $\mathrm{CM}$ rich in flavonoid compounds has an antiviral effect. ${ }^{(123)}$ Radix Scutellariae and Radix Glycyrrhizae in MXSG exhibit potential antiviral effects by binding to ACE2 and $3 C L$ hydrolase. ${ }^{(124)}$ (2) Inhibition of inflammatory storm and regulation immune function. RDN possesses the effect of regulating the activation of IL-6, IL-8, and TNF- $\alpha$, which protects COVID-19 patients from lung injury. ${ }^{(125)}$ XBJ plays a prominent role in anti-inflammation and has good immunostimulatory activity. ${ }^{(126)}$ (3) Inhibition of thrombosis. Some studies have shown that MXSG exerts the effect in increasing the solubility of ephedrine, which can inhibit platelet adhesion and aggregation in Ephedra. ${ }^{(124,127)}$ (4) Inhibition of oxidative stress. Emerging evidence indicated that glycyrrhizic acid has a good effect on anti-oxidative stress, inhibition of inflammatory reaction, and immunoregulation. ${ }^{(128)}$

\section{Conclusions and Prospects}

Owing to direct viral injury, endothelial damage and thrombosis, immune dysregulation, and RAAS overactivation, patients with severe COVID-19 might experience multiple organ complications or even die. Therefore, early identification is crucial to decrease the severity of disease and mortality of patients. The combination of CM and Western medicine is an efficient therapeutic strategy in the fight against COVID-19, which can improve clinical efficacy, delay progression of mild and moderate to severe and critical, and reduce the mortality rate. $\mathrm{CM}$ has won valuable time for the rehabilitation of severe types of patients. Currently, studies on CM mainly focus on the theory of CM and network pharmacology. In the future, large-scale, multicenter clinical studies, as well as pharmacological researches, should be carried out to systematically elaborate the efficacy of $\mathrm{CM}$.

To date, the mortality rate of COVID-19 patients has been greatly reduced. In the severe pandemic, scientists have recognized the molecular biological, 
and genetic of coronavirus, however, there are some unknowns about the coronavirus. With the continuously increasing understanding of pathogenesis, the maturity of treatment methods, and the popularization of vaccines, we hope to bring COVID-19 under control.

\section{Conflict of Interest}

The authors declare that they have no conflicts of interest.

\section{Author Contributions}

Wang XB was responsible for the design of this paper. Shi $K$ and Liu $Y$ drafted the manuscript and contributed equally to this work. Zhang Q, Ran CP, Hou J, and Zhang $Y$ were responsible for collecting information. Wang $X B$ revised and commented on the manuscript. All authors read and approved the final version for publication.

\section{Acknowledgements}

The authors are grateful to ZHAO Ting for her constructive comments in improving the language, grammar, and readability of the paper.

Electronic Supplementary Material: Supplementary material (Appendix 1) is available in the online version of this article at DOI: https://doi.org/10.1007/s11655-021-3313-x.

\section{REFERENCES}

1. Xiong $\mathrm{X}$, Wang $\mathrm{P}$, Su $\mathrm{K}$, et al. Chinese herbal medicine for coronavirus disease 2019: a systematic review and meta-analysis. Pharmacol Res 2020;160:105056.

2. Huang $\mathrm{C}$, Wang $\mathrm{Y}$, $\mathrm{Li} X$, et al. Clinical features of patients infected with 2019 novel coronavirus in Wuhan, China. Lancet 2020;395:497-506.

3. Marian AJ. Current state of vaccine development and targeted therapies for COVID-19: impact of basic science discoveries. Cardiovasc Pathol 2021;50:107278.

4. Wouters OJ, Shadlen KC, Salcher-Konrad M, et al. Challenges in ensuring global access to COVID-19 vaccines: production, affordability, allocation, and deployment. Lancet 2021;397:1023-1034.

5. Shin MD, Shukla S, Chung YH, et al. COVID-19 vaccine development and a potential nanomaterial path forward. Nat Nanotechnol 2020;15:646-655.

6. Gupta A, Madhavan MV, Sehgal K, et al. Extrapulmonary manifestations of COVID-19. Nat Med 2020;26:1017-1032.

7. Joly BS, Siguret V, Veyradier A. Understanding pathophysiology of hemostasis disorders in critically ill patients with COVID-19. Intens Care Med 2020;46:1603-1606.

8. Zeng $\mathrm{Y}$, Zhang B, Zhang $\mathrm{X}$, et al. Clinical characteristics of 9 cancer patients with SARS-CoV-2 infection. Chin Med 2020;15:47.

9. Liu J, Liu Y, Xiang P, et al. Neutrophil-to-lymphocyte ratio predicts critical illness patients with 2019 coronavirus disease in the early stage. J Transl Med 2020;18:206.

10. Phua J, Weng L, Ling L, et al. Intensive care management of coronavirus disease 2019 (COVID-19): challenges and recommendations. Lancet Respir Med 2020;8:506-517.

11. Hoffmann M, Kleine-Weber $\mathrm{H}$, Schroeder S, et al. SARS-CoV-2 cell entry depends on ACE2 and TMPRSS2 and is blocked by a clinically proven protease inhibitor. Cell 2020;181:271-280.

12. Bourgonje AR, Abdulle AE, Timens W, et al. Angiotensin-converting enzyme 2 (ACE2), SARS-CoV-2 and the pathophysiology of coronavirus disease 2019 (COVID-19). J Pathol 2020;251:228-248.

13. Qi F, Qian S, Zhang S, et al. Single cell RNA sequencing of 13 human tissues identify cell types and receptors of human coronaviruses. Biochem Biophys Res Commun 2020;526:135-140.

14. Pan $X W, X u D$, Zhang $H$, et al. Identification of a potential mechanism of acute kidney injury during the COVID-19 outbreak: a study based on single-cell transcriptome analysis. Intens Care Med 2020;46:1114-1116.

15. Ziegler CGK, Allon SJ, Nyquist SK, et al. SARS-CoV-2 receptor ACE2 is an interferon-stimulated gene in human airway epithelial cells and is detected in specific cell subsets across tissues. Cell 2020;181:1016-1035.

16. Leung JM, Yang CX, Tam A, et al. ACE-2 expression in the small airway epithelia of smokers and COPD patients: implications for COVID-19. Eur Respir J 2020;55:2000688.

17. Wölfel R, Corman VM, Guggemos W, et al. Virological assessment of hospitalized patients with COVID-2019. Nature 2020;581:465-469.

18. Teuwen LA, Geldhof V, Pasut A, et al. COVID-19: the vasculature unleashed. Nat Rev Immunol 2020;20:389-391.

19. Matacic C. Blood vessel injury may spur disease's fatal second phase. Science 2020;368:1039-1040.

20. Noris M, Benigni A, Remuzzi G. The case of complement activation in COVID-19 multiorgan impact. Kidney Int 2020;98:314-322.

21. McFadyen JD, Stevens H, Peter K. The emerging threat of (Micro) thrombosis in COVID-19 and its therapeutic implications. Circ Res 2020;127:571-587.

22. Moreno-Eutimio MA, López-Macías C, Pastelin-Palacios R. Bioinformatic analysis and identification of single-stranded RNA sequences recognized by TLR7/8 in the SARS-CoV-2, SARS-CoV, and MERS-CoV genomes. Microbes Infect 2020;22:226-229.

23. Foley $\mathrm{JH}$, Conway EM. Cross talk pathways between coagulation and inflammation. Circ Res 2016;118:1392-1408.

24. Magro C, Mulvey JJ, Berlin D, et al. Complement associated microvascular injury and thrombosis in the pathogenesis of severe COVID-19 infection: a report of five cases. Transl Res 2020;220:1-13.

25. Engelmann B, Massberg S. Thrombosis as an intravascular effector of innate immunity. Nat Rev Immunol 2013;13:34-45.

26. Koupenova M, Corkrey HA, Vitseva O, et al. The role of platelets in mediating a response to human influenza infection. Nat Commun 2019;10:1780.

27. Barnes BJ, Adrover JM, Baxter-Stoltzfus A, et al. Targeting potential drivers of COVID-19: neutrophil extracellular traps. J Exp Med 2020;217:e20200652.

28. Jung CJ, Yeh CY, Hsu RB, et al. Endocarditis pathogen promotes vegetation formation by inducing intravascular neutrophil extracellular traps through activated platelets. Circulation 2015;131:571-581.

29. Hu B, Huang S, Yin L. The cytokine storm and COVID-19. J Med Virol 2021;93:250-256.

30. Koupenova M, Clancy L, Corkrey HA, et al. Circulating platelets as mediators of immunity, inflammation, and thrombosis. Circ Res 2018;122:337-351.

31. Assinger A. Platelets and infection-an emerging role of platelets in viral infection. Front Immunol 2014;5:649.

32. Zhou F, Yu T, Du R, et al. Clinical course and risk factors for mortality of adult inpatients with COVID-19 in Wuhan, China: a retrospective cohort study. Lancet 2020;395:1054-1062.

33. Wu C, Chen X, Cai Y, et al. Risk factors associated with acute respiratory distress syndrome and death in patients with coronavirus disease 2019 pneumonia in Wuhan, China. JAMA Intern Med 2020;180:934-943.

34. Ruan Q, Yang K, Wang W, et al. Clinical predictors of mortality due to COVID-19 based on an analysis of data of 150 patients from Wuhan, China. Intens Care Med 2020;46:846-848.

35. Kayal S, Jaïs JP, Aguini N, et al. Elevated circulating E-selectin, intercellular adhesion molecule 1, and von Willebrand factor in patients with severe infection. Am J Respir Crit Care Med 1998;157:776-784.

36. Zhang $\mathrm{W}$, Zhao $\mathrm{Y}$, Zhang $\mathrm{F}$, et al. The use of anti-inflammatory drugs in the treatment of people with severe coronavirus disease 2019 (COVID-19): the perspectives of clinical immunologists from China. Clin 
Immunol 2020;214:108393.

37. Arachchillage DRJ, Laffan M. Abnormal coagulation parameters are associated with poor prognosis in patients with novel coronavirus pneumonia. J Thromb Haemost 2020;18:1233-1234.

38. Klok FA, Kruip M, van der Meer NJM, et al. Incidence of thrombotic complications in critically ill ICU patients with COVID-19. Thromb Res 2020;191:145-147.

39. Clerkin KJ, Fried JA, Raikhelkar J, et al. COVID-19 and cardiovascular disease. Circulation 2020;141:1648-1655.

40. Huertas A, Montani D, Savale L, et al. Endothelial cell dysfunction: a major player in SARS-CoV-2 infection (COVID-19)? Eur Respir J 2020;56:2001634.

41. Pober JS, Sessa WC. Evolving functions of endothelial cells in inflammation. Nat Rev Immunol 2007;7:803-815.

42. Jose RJ, Manuel A. COVID-19 cytokine storm: the interplay between inflammation and coagulation. Lancet Respir Med 2020;8:e46-e47.

43. Gupta N, Zhao YY, Evans CE. The stimulation of thrombosis by hypoxia. Thromb Res 2019;181:77-83.

44. Grimmer B, Kuebler WM. The endothelium in hypoxic pulmonary vasoconstriction. J Appl Physiol 2017;123:1635-1646.

45. Mehta P, McAuley DF, Brown M, et al. COVID-19: consider cytokine storm syndromes and immunosuppression. Lancet 2020;395:1033-1034.

46. Merad M, Martin JC. Pathological inflammation in patients with COVID-19: a key role for monocytes and macrophages. Nat Rev Immunol 2020;20:355-362.

47. Wang D, Hu B, Hu C, et al. Clinical characteristics of 138 hospitalized patients with 2019 novel coronavirus-infected pneumonia in Wuhan, China. JAMA 2020;323:1061-1069.

48. Petrilli CM, Jones SA, Yang J, et al. Factors associated with hospital admission and critical illness among 5279 people with coronavirus disease 2019 in New York City: prospective cohort study. BMJ 2020;369:m1966.

49. Kim KD, Zhao J, Auh S, et al. Adaptive immune cells temper initial innate responses. Nat Med 2007;13:1248-1252.

50. Lin L, Lu L, Cao W, et al. Hypothesis for potential pathogenesis of SARS-CoV-2 infection-a review of immune changes in patients with viral pneumonia. Emerg Microbes Infect 2020;9:727-732.

51. Kaplanski G, Marin V, Fabrigoule M, et al. Thrombin-activated human endothelial cells support monocyte adhesion in vitro following expression of intercellular adhesion molecule-1 (ICAM-1; CD54) and vascular cell adhesion molecule-1 (VCAM-1; CD106). Blood 1998;92:1259-1267.

52. Chen D, Carpenter A, Abrahams J, et al. Protease-activated receptor 1 activation is necessary for monocyte chemoattractant protein 1-dependent leukocyte recruitment in vivo. J Exp Med 2008;205:1739-1746.

53. Hanff TC, Harhay MO, Brown TS, et al. Is there an association between COVID-19 mortality and the renin-angiotensin system? a call for epidemiologic investigations. Clin Infect Dis 2020;71:870-874.

54. Ingraham NE, Barakat AG, Reilkoff R, et al. Understanding the renin-angiotensin-aldosterone-SARS-CoV axis: a comprehensive review. Eur Respir J 2020;56:2000912.

55. Ghazi L, Drawz P. Advances in understanding the renin-angiotensinaldosterone system (RAAS) in blood pressure control and recent pivotal trials of RAAS blockade in heart failure and diabetic nephropathy. F1000Res 2017;6:F1000 Faculty Rev-297.

56. Voors AA, Pinto YM, Buikema $\mathrm{H}$, et al. Dual pathway for angiotensin II formation in human internal mammary arteries. $\mathrm{Br} \mathrm{J}$ Pharmacol 1998;125:1028-1032.

57. Zhang $\mathrm{H}$, Penninger JM, Li Y, et al. Angiotensin-converting enzyme 2 (ACE2) as a SARS-CoV-2 receptor: molecular mechanisms and potential therapeutic target. Intens Care Med 2020;46:586-590.

58. Verdecchia $P$, Cavallini $C$, Spanevello $A$, et al. The pivotal link between ACE2 deficiency and SARS-CoV-2 infection. Eur J Intern Med 2020;76:14-20.

59. Gheblawi M, Wang K, Viveiros A, et al. Angiotensin-converting enzyme 2: SARS-CoV-2 receptor and regulator of the renin-angiotensin system: celebrating the 20th Anniversary of the discovery of ACE2. Circ Res 2020;126:1456-1474.

60. Patel VB, Clarke N, Wang Z, et al. Angiotensin II induced proteolytic cleavage of myocardial ACE2 is mediated by TACE/ ADAM-17: a positive feedback mechanism in the RAS. J Mol Cell Cardiol 2014;66:167-176.

61. Miao CX, Wang B, Wang $\mathrm{Y}$, et al. Understanding and thinking of novel coronavirus pneumonia in traditional Chinese medicine. $J$ Tradit Chin Med 2020;61:286-288.

62. Tong $L X$, Zhao $L$, Li Q, et al. Discussion on traditional Chinese medicine prevention and treatment strategies of Coronavirus Disease 2019 (COVID-19) from the perspective of "cold-dampness pestilence". J Tradit Chin Med 2020;61:465-470.

63. Wang QW, Ma J, Ruan L, et al. Clinical features, and syndrome differentiation of novel coronavirus pneumonia in traditional Chinese medicine. J Tradit Chin Med 2020;61:281-285.

64. Yang LD, Chao $E$, Zhang $H$. Thinking on etiology and pathogenesis of novel coronavirus pneumonia in traditional Chinese medicine. $\mathrm{J}$ Tradit Chin Med 2020;61:557-560.

65. Feng $\mathrm{Y}$, Ling $\mathrm{Y}$, Bai $\mathrm{T}$, et al. COVID-19 with different severities: a multicenter study of clinical features. Am J Respir Crit Care Med 2020;201:1380-1388.

66. Li X, Xu S, Yu M, et al. Risk factors for severity and mortality in adult COVID-19 inpatients in Wuhan. J Allergy Clin Immunol 2020;146:110-118.

67. Louapre C, Collongues N, Stankoff B, et al. Clinical characteristics and outcomes in patients with Coronavirus Disease 2019 and multiple sclerosis. JAMA Neurol 2020;77:1079-1088.

68. Robilotti EV, Babady NE, Mead PA, et al. Determinants of COVID-19 disease severity in patients with cancer. Nat Med 2020;26:1218-1223.

69. Yang K, Sheng Y, Huang C, et al. Clinical characteristics, outcomes, and risk factors for mortality in patients with cancer and COVID-19 in Hubei, China: a multicentre, retrospective, cohort study. Lancet Oncol 2020;21:904-913.

70. Grasselli G, Greco M, Zanella A, et al. Risk factors associated with mortality among patients with COVID-19 in intensive care units in Lombardy, Italy. JAMA Intern Med 2020;180:1345-1355.

71. Banerjee A, Pasea L, Harris S, et al. Estimating excess 1-year mortality associated with the COVID-19 pandemic according to underlying conditions and age: a population-based cohort study. Lancet 2020;395:1715-1725.

72. Gupta S, Hayek SS, Wang W, et al. Factors associated with death in critically ill patients with Coronavirus Disease 2019 in the US. JAMA Intern Med 2020;180:1-12.

73. Zheng $\mathrm{Y}$, Zhang $\mathrm{Y}$, Chi $\mathrm{H}$, et al. The hemocyte counts as a potential biomarker for predicting disease progression in COVID-19: a retrospective study. Clin Chem Lab Med 2020;58:1106-1115.

74. Chen G, Wu D, Guo W, et al. Clinical and immunological features of severe and moderate coronavirus disease 2019. J Clin Invest 2020;130:2620-2629.

75. Liang W, Yao J, Chen A, et al. Early triage of critically ill COVID-19 patients using deep learning. Nat Commun 2020;11:3543.

76. Wu G, Yang P, Xie Y, et al. Development of a clinical decision support system for severity risk prediction and triage of COVID-19 patients at hospital admission: an international multicentre study. Eur Respir J 2020;56:2001104.

77. Liang W, Liang $\mathrm{H}, \mathrm{Ou}$ L, et al. Development and validation of a clinical risk score to predict the occurrence of critical illness in hospitalized patients with COVID-19. JAMA Intern Med 2020;180:1081-1089.

78. Al-Samkari H, Karp Leaf RS, Dzik WH, et al. COVID-19 and coagulation: bleeding and thrombotic manifestations of SARSCoV-2 infection. Blood 2020;136:489-500.

79. Mazzoni A, Salvati L, Maggi L, et al. Impaired immune cell cytotoxicity in severe COVID-19 is IL-6 dependent. J Clin Invest 2020;130:4694-4703.

80. Herold T, Jurinovic V, Arnreich C, et al. Elevated levels of IL-6 and CRP predict the need for mechanical ventilation in COVID-19. J Allergy Clin Immunol 2020;146:128-136.

81. Tian J, Yuan X, Xiao J, et al. Clinical characteristics and risk factors associated with COVID-19 disease severity in patients with cancer in Wuhan, China: a multicentre, retrospective, cohort study. Lancet 
Oncol 2020;21:893-903

82. McElvaney OJ, McEvoy NL, McElvaney OF, et al. Characterization of the inflammatory response to severe COVID-19 illness. Am J Respir Crit Care Med 2020;202:812-821.

83. Zhang $X$, Tan $Y$, Ling $Y$, et al. Viral and host factors related to the clinical outcome of COVID-19. Nature 2020;583:437-440.

84. Yang Y, Shen C, Li J, et al. Plasma IP-10 and MCP-3 levels are highly associated with disease severity and predict the progression of COVID-19. J Allergy Clin Immunol 2020;146:119-127.

85. Shen B, Yi X, Sun Y, et al. Proteomic and metabolomic characterization of COVID-19 patient sera. Cell 2020;182:59-72.

86. Song JW, Zhang C, Fan X, et al. Immunological and inflammatory profiles in mild and severe cases of COVID-19. Nat Commun 2020;11:3410.

87. Hadjadj J, Yatim N, Barnabei L, et al. Impaired type I interferon activity and inflammatory responses in severe COVID-19 patients. Science 2020;369:718-724.

88. Kong $\mathrm{Y}, \mathrm{Han} \mathrm{J}, \mathrm{Wu} \mathrm{X}$, et al. VEGF-D: a novel biomarker for detection of COVID-19 progression. Crit Care 2020;24:373.

89. Grein J, Ohmagari N, Shin D, et al. Compassionate use of remdesivir for patients with severe Covid-19. N Engl J Med 2020;382:2327-2336.

90. Ko WC, Rolain JM, Lee NY, et al. Arguments in favour of remdesivir for treating SARS-CoV-2 infections. Int J Antimicrob Agents 2020;55:105933.

91. Wang $Y$, Zhang D, Du G, et al. Remdesivir in adults with severe COVID-19: a randomised, double-blind, placebo-controlled, multicentre trial. Lancet 2020;395:1569-1578.

92. Siemieniuk RA, Bartoszko JJ, Ge L, et al. Drug treatments for Covid-19: living systematic review and network meta-analysis. BMJ 2020;370:m2980

93. Drożdżal S, Rosik J, Lechowicz K, et al. FDA approved drugs with pharmacotherapeutic potential for SARS-CoV-2 (COVID-19) therapy. Drug Resist Updat 2020;53:100719.

94. Wise J. Covid-19: remdesivir is recommended for authorisation by European Medicines Agency. BMJ 2020;369:m2610.

95. Stauffer WM, Alpern JD, Walker PF. COVID-19 and dexamethasone: a potential strategy to avoid steroid-related strongyloides hyperinfection. JAMA 2020; 324:623-624.

96. Mahase E. Covid-19: low dose steroid cuts death in ventilated patients by one third, trial finds. BMJ 2020;369:m2422.

97. Horby P, Lim WS, Emberson JR, et al. Dexamethasone in hospitalized patients with Covid-19. N Engl J Med 2021;384:693-704.

98. Villar J, Ferrando C, Martínez D, et al. Dexamethasone treatment for the acute respiratory distress syndrome: a multicentre, randomised controlled trial. Lancet Respir Med 2020;8:267-276.

99. Voicu S, Bonnin P, Stépanian A, et al. High prevalence of deep vein thrombosis in mechanically ventilated COVID-19 patients. J Am Coll Cardiol 2020;76:480-482.

100. Shi W, Lv J, Lin L. Coagulopathy in COVID-19: focus on vascular thrombotic events. J Mol Cell Cardiol 2020;146:32-40.

101. Levi M, Thachil J, Iba T, et al. Coagulation abnormalities and thrombosis in patients with COVID-19. Lancet Haematol 2020;7:e438-e440.

102. Bikdeli B, Madhavan MV, Jimenez D, et al. COVID-19 and thrombotic or thromboembolic disease: implications for prevention, antithrombotic therapy, and follow-up: JACC state-of-the-art review. J Am Coll Cardiol 2020;75:2950-2973.

103. Tang N, Bai $\mathrm{H}$, Chen $\mathrm{X}$, et al. Anticoagulant treatment is associated with decreased mortality in severe coronavirus disease 2019 patients with coagulopathy. J Thromb Haemost 2020;18:1094-1099.

104. Helms J, Tacquard C, Severac F, et al. High risk of thrombosis in patients with severe SARS-CoV-2 infection: a multicenter prospective cohort study. Intensive Care Med 2020;46:1089-1098.

105. Cheng $\mathrm{Y}$, Wong $\mathrm{R}$, Soo $\mathrm{YO}$, et al. Use of convalescent plasma therapy in SARS patients in Hong Kong. Eur J Clin Microbiol Infect Dis 2005; 24:44-46.

106. Hung IF, To KK, Lee CK, et al. Convalescent plasma treatment reduced mortality in patients with severe pandemic influenza $A$ (H1N1) 2009 virus infection. Clin Infect Dis 2011;52:447-456.

107. Ko JH, Seok H, Cho SY, et al. Challenges of convalescent plasma infusion therapy in Middle East respiratory coronavirus infection: a single centre experience. Antivir Ther 2018;23:617-622.

108. Shen $C$, Wang Z, Zhao F, et al. Treatment of 5 critically ill patients with COVID-19 with convalescent plasma. JAMA 2020;323:1582-1589.

109. Duan K, Liu B, Li C, et al. Effectiveness of convalescent plasma therapy in severe COVID-19 patients. Proc Natl Acad Sci U S A 2020;117:9490-9496.

110. Tanne JH. Covid-19: FDA approves use of convalescent plasma to treat critically ill patients. BMJ 2020;368:m1256.

111. Luo H, Gao Y, Zou J, et al. Reflections on treatment of COVID-19 with traditional Chinese medicine. Chin Med 2020;15:94

112. Zhang D, Zhang B, Lv JT, et al. The clinical benefits of Chinese patent medicines against COVID-19 based on current evidence. Pharmacol Res 2020;157:104882.

113. Li C, Wang L, Ren L. Antiviral mechanisms of candidate chemical medicines and traditional Chinese medicines for SARS-CoV-2 infection. Virus Res 2020;286:198073.

114. Wang JB, Wang ZX, Jing J, et al. Exploring an integrative therapy for treating COVID-19: a randomized controlled trial. Chin J Integr Med 2020;26:648-655.

115. Chen G, Su W, Yang J, et al. Chinese herbal medicine reduces mortality in patients with severe and critical Coronavirus disease 2019: a retrospective cohort study. Front Med 2020;14:752-759.

116. Wang $Y$, Liu Y, Lv Q, et al. Effect and safety of Chinese herbal medicine granules in patients with severe coronavirus disease 2019 in Wuhan, China: a retrospective, single-center study with propensity score matching. Phytomedicine 2020;85:153404.

117. Yang R, Liu H, Bai C, et al. Chemical composition and pharmacological mechanism of Qingfei Paidu Decoction and Ma Xing Shi Gan Decoction against Coronavirus Disease 2019 (COVID-19): in silico and experimental study. Pharmacol Res 2020;157:104820.

118. Lee DYW, Li QY, Liu J, et al. Traditional Chinese herbal medicine at the forefront battle against COVID-19: clinical experience and scientific basis. Phytomedicine 2021;80:153337.

119. Shi N, Liu B, Liang N, et al. Association between early treatment with Qingfei Paidu Decoction and favorable clinical outcomes in patients with COVID-19: a retrospective multicenter cohort study. Pharmacol Res 2020;161:105290.

120. Huang L. Efficacy and safety assessment of severe COVID-19 patients with Chinese medicine: a retrospective case series study at early stage of the COVID-19 epidemic in Wuhan, China. J Ethnopharmacol 2021;277:113888.

121. Liu J, Jiang Y, Liu Y, et al. Yindan Jiedu Granules, a traditional Chinese medicinal formulation, as a potential treatment for coronavirus disease 2019. Front Pharmacol 2020;11:634266.

122. Guo H, Zheng J, Huang G, et al. Xuebijing Injection in the treatment of COVID-19: a retrospective case-control study. Ann Palliat Med 2020;9:3235-3248.

123. LiRF, Hou YL, Huang JC, et al. Lianhuaqingwen exerts anti-viral and anti-inflammatory activity against novel coronavirus (SARS-CoV-2). Pharmacol Res 2020;156:104761.

124. Ren X, Shao XX, Li XX, et al. Identifying potential treatments of COVID-19 from traditional Chinese medicine (TCM) by using a datadriven approach. J Ethnopharmacol 2020;258:112932.

125. Jia S, Luo H, Liu X, et al. Dissecting the novel mechanism of reduning injection in treating Coronavirus Disease 2019 (COVID-19) based on network pharmacology and experimental verification. J Ethnopharmacol 2021;273:113871.

126. Chen S, Dai G, Hu J, et al. Discovery of Xuebijing Injection exhibiting protective efficacy on sepsis by inhibiting the expression of HMGB1 in septic rat model designed by cecal ligation and puncture. Am J Ther 2016;23:e1819-e1825.

127. Flordal PA, Svensson J. Hemostatic effects of ephedrine. Thromb Res 1992;68:295-302.

128. Li X, Sun R, Liu R. Natural products in licorice for the therapy of liver diseases: progress and future opportunities. Pharmacol Res 2019;144:210-226.

(Accepted April 16, 2021) Edited by TIAN Lin 